\title{
Nutrição parenteral com foco na composição lipídica: uma breve revisão
}

\author{
Parenteral nutrition with a focus on lipid composition: a brief review \\ Nutrición parenteral con enfoque en la composición lípidica: una breve revisión
}

Recebido: 25/01/2022 | Revisado: 01/02/2022 | Aceito: 17/02/2022 | Publicado: 25/02/2022

\author{
Marciele Alves Bolognese \\ ORCID: https://orcid.org/0000-0003-3417-9566 \\ Universidade Estadual de Maringá, Brasil \\ E-mail: mafb-2006@hotmail.com \\ Patrícia Magalhães de Souza \\ ORCID: https://orcid.org/0000-0001-5916-0744 \\ Universidade Estadual de Maringá, Brasil \\ E-mail: patricia.magalhaes11@hotmail.com \\ Vanessa Javera Castanheira Néia \\ ORCID: https://orcid.org/0000-0003-2573-3457 \\ Universidade Estadual de Maringá, Brasil \\ E-mail: nutrivanjavera@hotmail.com \\ Oscar Oliveira Santos \\ ORCID: https://orcid.org/0000-0002-9631-8480 \\ Universidade Estadual de Maringá, Brasil \\ E-mail: oliveirasantos.oscardeoliveira@gmail.com \\ Jesuí Vergílio Visentainer \\ ORCID: https://orcid.org/0000-0003-3412-897X \\ Universidade Estadual de Maringá, Brasil \\ E-mail: jesuivv@gmail.com
}

\begin{abstract}
Resumo
A nutrição parenteral (NP) tem como objetivo o fornecimento de uma mistura de nutrientes aos pacientes em tratamento, por meio do sistema venoso, a fim de restituir seu aporte calórico e evitar complicações. Neste caso, os lipídios desempenham papel significativo devido as suas funções biológicas e sua alta densidade energética que o torna apto para a recuperação do aporte calórico do paciente em tratamento. Na NP, as emulsões lipídicas são elaboradas a base de alimentos ricos em ácidos graxos essenciais, como óleos vegetais, em especial, o óleo de soja, o qual é fonte majoritária do ácido graxo ômega 6, azeite de oliva (rico em ácido oleico), e óleo de peixe, fonte de EPA e DHA. O emprego desses ácidos graxos corrobora para a completa manutenção da saúde. Logo, o objetivo do presente trabalho foi elaborar uma revisão narrativa acerca dos benefícios dos lipídios na recuperação dos pacientes, submetidos a terapia nutricional parenteral. Para o fichamento dos principais manuscritos sobre o tema em questão, utilizou-se plataformas incluindo a Web of Science, Google Scholar, Scopus, PubMed, SciELO e Portal Periodicos Capes. Os resultados obtidos auxiliam os profissionais da área a fornecerem aos seus pacientes a terapia de emulsão lipídica mais adequada visando a melhora do seu quadro clínico.
\end{abstract}

Palavras-chave: Terapia nutricional parenteral; Emulsão lipídica; Lipídios; Ácidos graxos; Ômega 3; Ômega 6.

\begin{abstract}
Parenteral nutrition $(\mathrm{PN})$ aims to provide a mixture of nutrients to patients undergoing treatment, through the venous system, in order to restore their caloric intake and avoid complications. In this case, lipids play a significant role due to their biological functions and their high energy density, which makes them suitable for the recovery of the patient's caloric intake. In NP, lipid emulsions are made from foods rich in essential fatty acids, such as vegetable oils, especially soybean oil, which is a major source of omega-6 fatty acid, olive oil (rich in oleic acid), and fish oil, source of EPA and DHA. The use of these fatty acids contributes to the complete maintenance of health. Therefore, the objective of the present study was to elaborate a narrative review about the benefits of lipids in the recovery of patients undergoing parenteral nutritional therapy. Platforms including the Web of Science, Google Scholar, Scopus, PubMed, SciELO and Portal Periodicos Capes were used to file the main manuscripts on the subject in question. The results obtained help professionals in the field to provide their patients with the most appropriate lipid emulsion therapy to improve their clinical condition.
\end{abstract}

Keywords: Parenteral nutritional therapy; Lipid emulsion; Lipids; Fatty acids; Omega 3; Omega 6. 


\section{Resumen}

La nutrición parenteral (NP) tiene como objetivo proporcionar una mezcla de nutrientes a los pacientes en tratamiento, a través del sistema venoso, con el fin de restablecer su aporte calórico y evitar complicaciones. En este caso, los lípidos juegan un papel importante debido a sus funciones biológicas y su alta densidad energética, lo que los hace idóneos para la recuperación del aporte calórico del paciente. En NP, las emulsiones lipídicas se elaboran a partir de alimentos ricos en ácidos grasos esenciales, como los aceites vegetales, especialmente el aceite de soja, que es una fuente importante de ácidos grasos omega-6, el aceite de oliva (rico en ácido oleico) y el aceite de pescado, fuente de EPA y DHA. El uso de estos ácidos grasos contribuye al mantenimiento completo de la salud. Por lo tanto, el objetivo del presente estudio fue elaborar una revisión narrativa sobre los beneficios de los lípidos en la recuperación de pacientes en tratamiento nutricional parenteral. Se utilizaron plataformas como Web of Science, Google Scholar, Scopus, PubMed, SciELO y Portal Periódicos Capes para registrar los principales manuscritos sobre el tema en cuestión. Los resultados obtenidos ayudan a los profesionales del sector a proporcionar a sus pacientes la terapia de emulsión lipídica más adecuada para mejorar su estado clínico.

Palabras clave: Terapia nutricional parenteral; Emulsión de lípidos; Lípidos; Ácidos grasos; Omega 3; Omega 6.

\section{Introdução}

No âmbito da terapia nutricional, a nutrição parenteral (NP) tem por objetivo fornecer uma mistura de nutrientes aos pacientes em tratamento de algumas doenças crônicas, ou até mesmo hospitalizados, por meio do sistema venoso, com o intuito de restituir seu aporte calórico e evitar complicações (Torrinhas \& Waitzberg, 2016). A escolha pela NP se dá, a partir do momento em que não é possível, ou seria inseguro utilizar outras vias como a oral e a enteral, devido a condição do paciente e da função de seu sistema gastrointestinal (Berger \& Pichard, 2022).

As dietas parenterais são compostas por macronutrientes e micronutrientes incluindo, carboidratos, aminoácidos, eletrólitos, vitaminas, minerais e lipídios (Cristina, 2009; Martindale et al., 2020; Versleijen et al., 2005). Dentre os componentes citados, os lipídios são importantes na NP, por estar envolvido em inúmeras funções biológicas e metabólicas. Ademais, sua alta densidade energética fornece aporte calórico para pacientes que estão em tratamento (Carpentier et al., 2000; Souza \& Visentainer, 2006; Versleijen et al., 2005).

Na NP, a classe lipídica mais relatada são os triacilgliceróis (TAGs), especialmente na formulação de emulsões parenterais lipídicas, isso porque são os principais fornecedores de energia ao ser humano, bem como de ácidos graxos saturados (AGS), monoinsaturados (AGMI), e poli-insaturados (AGPI) (Calder \& Jensen, 2010; Raman et al., 2017; Souza \& Visentainer, 2006).

As emulsões lipídicas para a NP apresentam alta densidade energética, baixa osmolaridade e consequentemente, inúmeros benefícios aos pacientes (Adolph, 1999; Klek, 2016; Raman et al., 2017; Singer et al., 2019). Essas emulsões são elaboradas à base de alimentos ricos em AGs estritamente essenciais, como óleos vegetais, em especial, o óleo de soja, o qual é fonte majoritária do AGs ômega 6 ( n-6), e também o óleo de peixe, fonte de ácido Eicosapentaenóico (EPA) e ácido Docosahexaenoico (DHA) (Calder, 2012; Klek, 2016; Martindale et al., 2020).

Dessa forma, o objetivo do presente trabalho é elaborar uma breve revisão narrativa acerca dos benefícios dos lipídios na recuperação dos pacientes, submetidos a terapia nutricional parenteral.

\section{Metodologia}

A pesquisa refere-se a uma revisão narrativa da literatura, a qual possui caráter amplo, e se propõe a descrever sobre a terapia nutricional parenteral com foco na composição lipídica, sob o ponto de vista teórico e contextual, mediante análise e interpretação dos manuscritos escolhidos.

A síntese de conhecimentos do tema em questão, se faz importante, visto que favorece o campo da pesquisa científica, fornecendo subsídio para elaboração de novos trabalhos, além de aumentar o conhecimento do leitor. Assim, para o fichamento 
dos principais manuscritos sobre o tema em questão, utilizou-se plataformas incluindo a Web of Science, Google Scholar, Scopus, PubMed, SciELO e Portal Periodicos Capes. Como critério para inclusão das publicações, escolheu-se palavras delimitadoras que apareceram nos resumos, títulos e palavras chaves, como nutrição parenteral lipídica, lipídios, AGs e nutrição parenteral, AGs poli-insaturados, importância dos AGs ômega 3 e ômega 6, emulsões lipídicas a base de óleo de soja, óleo de peixe e azeite de oliva, além de artigos publicados nos últimos 10 anos.

Posteriormente, realizou-se a leitura cuidadosa destes, com destaque para os tópicos mais relevantes, que subsidiaram a elaboração do conteúdo proposto.

\section{Resultados e Discussão}

\subsection{Nutrição Parenteral}

$\mathrm{O}$ suporte nutricional na área de cuidados intensivos tem se mostrado um grande desafio para os profissionais de saúde ao longo dos anos. No entanto, devido aos avanços da pesquisa científica e da tecnologia na área da saúde, é possível uma melhor aceitação social, bem como uma riqueza de conteúdo e informações, o que levou a uma maior consolidação da terapia nutricional (Singer et al., 2009, 2019).

No contexto da terapia nutricional, a nutrição parenteral (NP) visa fornecer aos pacientes desnutridos ou em risco de desnutrição, uma mistura de nutrientes via sistema intravenoso para restabelecer sua ingestão calórica e evitar complicações (Torrinhas \& Waitzberg, 2016). A NP é uma opção quando há comprometimento do funcionamento do sistema gastrointestinal, ou quando outras vias (por exemplo, oral e enteral) não são seguras (Berger \& Pichard, 2022).

Historicamente, a NP surgiu em 1960 com a finalidade de tratar pacientes com caquexia (doença que envolve perda de peso marcante e de massa muscular), e/ou câncer no estado mais avançado. A princípio, a NP era conhecida como "hiperalimentação" devido seu uso somente em pacientes em estado grave de desnutrição. Porém, nos anos de 1980 e 1990, esta se difundiu e foi considerada uma alternativa promissora a nutrição enteral (NE), a qual era mais utilizada na época (Berger, 2014; Berger \& Pichard, 2022).

Devido ao recente surgimento da NP, muitos profissionais tiveram receio em aplicá-la e prescrevê-la aos pacientes, tanto que há relatos de que muitos agravamentos de quadros clínicos foram devido a "hiperalimentação", ou seja, oferta maior de determinado nutriente do que o organismo do paciente realmente necessitava. De fato, como tudo que é novo, esperava-se o surgimento de dúvidas, e até mesmo falhas. Atualmente, a NP é amplamente conhecida e aplicada nas terapias nutricionais e, mesmo com suas vantagens e desvantagens, ao longo dos anos, tal procedimento evolui e conquista seu espaço (Berger, 2014; Itzhaki \& Singer, 2020; Kojic et al., 2017).

\subsection{Composição Lipídica das Dietas Parenterais}

Uma dieta parenteral consiste em macronutrientes e micronutrientes incluindo carboidratos, aminoácidos, eletrólitos, vitaminas, minerais e lipídios. Dos componentes mencionados, os lipídios são importantes na NP por fornecer maior aporte energético (Cristina, 2009; Martindale et al., 2020; Versleijen et al., 2005)

Os lipídios são definidos como um amplo e complexo grupo de compostos orgânicos encontrados em organismos vivos e são solúveis em solventes orgânicos, e insolúveis em água. Os lipídios são classificados conforme sua polaridade: neutros e polares. Nos lipídios neutros estão inclusos os triacilgliceróis (TAGs), diacilglicerois (DAGs), monoacilgliceróis (MAGs), esteróis (principalmente colesterol livre e esterificado), ceras e ácidos graxos livres (AGL). Já para os lipídios polares tem-se os fosfolipídios (FL) e glicolipídios (GL) (Souza \& Visentainer, 2006).

$\mathrm{Na} \mathrm{NP}$, os lipídios são considerados de extrema importância, devido as suas funções biológicas, como armazenamento de energia, formação das membranas e sinalização celular. Ademais, sua alta densidade energética o torna apto para a 
recuperação do aporte calórico do paciente em tratamento (Carpentier et al., 2000; Souza \& Visentainer, 2006; Versleijen et al., 2005).

Dentre as classes lipídicas, os TAGs, são os mais utilizados na NP, visto que são os principais fornecedores de energia ao ser humano, bem como de ácidos graxos saturados (AGS), monoinsaturados (AGMI), e poli-insaturados (AGPI) (Calder \& Jensen, 2010; Raman et al., 2017; Souza \& Visentainer, 2006).

\subsection{1 Ácidos Graxos}

Os ácidos graxos (AGs) são ácidos carboxílicos representados pela fórmula RCOOH, onde o grupamento R pode ser uma cadeia carbônica curta, média ou longa.

Os AGs de cadeia curta apresentam de dois a quatro átomos de carbono, média de seis a dez átomos de carbono, e longa acima de doze átomos de carbono. Além disso, os AGs diferem-se pela presença ou não de ligações duplas. Todos esses fatores podem afetar suas propriedades biológicas e consequentemente influenciar em processos imunológicos e inflamatórios do paciente (Hewavitharana et al., 2020).

Os AGs são, de fato, importantes para a manutenção da saúde, visto que são os principais constituintes das membranas celulares, contribuindo para total integridade destas. Além de-serem precursores dos principais modulares envolvidos nas vias celulares do sistema imunológico, tais como citocinas e anticorpos (Perini et al., 2010).

$\mathrm{Na} N$, os AGs mais usados, são aqueles da série ômega 3 como o ácido alfa linolênico (C18:3 n-3); e os da série ômega 6 como o ácido linoleico (C18:2 n-6). Isso porque o emprego desses AGPIs na dieta está associado a benefícios à saúde como, manutenção das funções cerebrais, desenvolvimento das células, diminuição da pressão sanguínea, prevenção de enfermidades cardiovasculares, proteção contra feridas e infecções, dentre outros. Por essa razão, o consumo das dietas parenterais que contenham tais AGPs, se torna essencial (Barbalho et al., 2011; Calder et al., 2018; Ramalho \& Suarez, 2013; Raman et al., 2017).

Os AGPIs linoléico e alfa-linolênico são considerados estritamente essenciais, pois não são sintetizados pelo organismo humano; e nem por ácidos graxos precursores, portanto devem ser obtidos através da alimentação. Já os ácidos graxos que são sintetizados (derivados) a partir dos precursores ácidos linoléico e alfa-linolênico, são denominados ácidos graxos essenciais. Como principais representantes da série n-3, tem-se o ácido eicosapentaenoico (C20:5 n-3, EPA) e o ácido docosahexaenóico (C22:6 n-3, DHA), enquanto que para a série n-6, tem-se o ácido araquidônico (C20:4 n-6, AA) (Perini et al., 2010; Ramalho \& Suarez, 2013).

Durante anos, o papel dos AGPIs na modulação das atividades biológicas vem sendo estudado. Os primeiros trabalhos relatam que indivíduos acometidos por situação de risco que incluíram na sua dieta uma quantidade considerável de EPA e DHA, a possibilidade do aparecimento de doenças cardiovasculares foi menor, quando comparado a pessoas que não ingeriram tais AGPIs. No entanto, apesar dos benefícios associados ao consumo destes, deve-se tomar o devido cuidado com o equilíbrio dos AGs n-3 e n-6, visto que enquanto os AGs da série n-6 favorecem respostas inflamatórias, os AGs n-3, como EPA e DHA exercem menos ações pró-inflamatórias (Geert, 2007; Perini et al., 2010).

\subsection{Emulsões Lipídicas}

Na NP, os AGs são fornecidos aos pacientes por meio de emulsões lipídicas. As emulsões lipídicas para a NP foram estabelecidas há anos, devido a sua alta densidade energética, baixa osmolaridade, e inúmeros benefícios que ela fornece aos pacientes, dentre eles, a redução da dependência da dextrose, melhora do sistema imunológico e inflamatório, dentre outros (Adolph, 1999; Klek, 2016; Raman et al., 2017; Singer et al., 2019). 
Essas emulsões são elaboradas à base de alimentos ricos em AGs estritamente essenciais, como óleos vegetais, em especial, o óleo de soja, o qual é fonte majoritária do AGs n-6 e também o óleo de peixe, fonte de EPA e DHA (Calder, 2012; Klek, 2016; Martindale et al., 2020).

Uma grande variedade de emulsões lipídicas está disponível para uso na NP, com composições distintas em AGs, proteínas, carboidratos, vitaminas, entre outros, cabendo então a um profissional qualificado prescrever qual delas é mais adequada para fornecer os nutrientes que o paciente necessita. A diferença no fornecimento dos compostos da dieta, podem influenciar nas propriedades funcionais, incluindo a regulação da estrutura e função das membranas celulares, assim como na regulação das vias de sinalização intracelular, atividade do fator de transcrição, expressão gênica e regulação da produção de mediadores lipídicos bioativos. Ademais, o uso das emulsões lipídicas na NP pode possibilitar a melhora no quadro do paciente em estado crítico, por meio do melhor controle dos processos inflamatórios e biológicos (Calder et al., 2018; Calder \& Jensen, 2010; Carpentier et al., 2000; Krohn \& Koletzko, 2006).

\subsubsection{Emulsões Lipídicas à base de óleo de Soja, Azeite de Oliva e Óleo de peixe}

O óleo de soja tem sido tradicionalmente usado no preparo da emulsão lipídica para a NP, devido a sua composição rica em AGs insaturados (aproximadamente 85\% do total), sendo que os mais encontrados são: ácido linolênico (4-11\%), ácido oleico (19-30\%), e o ácido linoleico (44-62\%). O ácido linoleico é o mais presente na NP (Zhao et al., 2022).

Mesmo conhecendo os benefícios dos AGs da série n-6, o que justifica o uso do óleo de soja para a formulação de dietas, há uma grande preocupação quanto ao elevado consumo deste, devido ao aumento das chances de desenvolver doenças inflamatórias e insultos traumáticos. Estudos apontam que as emulsões lipídicas baseadas puramente em óleo de soja, devem ser evitadas. Por consequência, muitas alternativas de emulsões à base de outros alimentos (azeite de oliva, óleo de peixe) e até mesmo misturas (óleo de soja + aditivos) foram relatadas (Krohn \& Koletzko, 2006).

O azeite de oliva, apresenta diversas propriedades benéficas, provindas de suas características organolépticas e composição química, podendo,-ser utilizado para o tratamento de enfermidades, como por exemplo úlceras estomacais, além de problemas com desnutrição (Gavahian et al., 2019). A composição em AGs do azeite de oliva inclui, AGS (14,5\%), consistindo de $13 \%$ de ácido palmítico (16:0) e 1,5\% de ácido esteárico (18:0) e AGs insaturados (85\%), sendo que o ácido oleico (18:1n-9) o majoritário, representando cerca de 70\% da composição total. Em seguida, tem-se o ácido linoleico (18:2 n6) com composição próxima a 15\% e por fim o ácido palmitoléico (16:1 n-7), com 3,5\% (Rousseaux et al., 2020).

Observa-se que o ácido oleico é o principal AGs fornecido pelo azeite, isso é de fato vantajoso no preparo das emulsões lipídicas, visto que o mesmo é conhecido pelo seu menor impacto potencial na função imunológica, inflamação e coagulação sanguínea quando comparado aos AGs n-6 por exemplo (Perini et al., 2010; Raman et al., 2017).

Torrinhas \& Waitzberg (2016) afirmam que o uso de elevadas quantidades de AGPIs nas formulações parenterais, podem acarretar reações indesejáveis de peroxidação lipídica, enquanto o mesmo não ocorre para-dietas ricas em AGMIs como nas emulsões feitas a partir do azeite de oliva. Acredita-se portanto, que tal produto seja menos suscetível ao estresse oxidativo quando comparado ao óleo de soja nas mesmas condições, por essa razão, o uso de emulsões lipídicas à base de azeite de oliva é uma possibilidade (Casselman et al., 2021; Torrinhas \& Waitzberg, 2016).

Outro tipo de emulsão amplamente utilizada, é a preparada com óleo de peixe, isso porque ele é rico em AGPIs de cadeia longa, (EPA e DHA). Ambos AGPIs exercem benefícios ao paciente em tratamento, sobretudo de enfermidades cardiovasculares, doenças anti-inflamatórias, gastrointestinais, infecções, além da prevenção de lesões, alterações imunológicas e coagulação sanguínea, (Chen \& Yeh, 2003; Novak et al., 2019). Assim, afirma-se que o óleo de peixe em particular, pode ser uma alternativa para substituir, o óleo de soja como componente da nutrição parenteral (Boisramé-helms et al., 2015; Chen \& Yeh, 2003). 


\section{Considerações Finais}

Com base no presente trabalho, foi possível destacar as principais informações acerca da terapia nutricional parenteral com foco na composição lipídica, sob o ponto de vista teórico e contextual, evidenciando a importância deste tema, e incentivando pesquisas futuras, e principalmente, ampliar o conhecimento do leitor. Ainda, os resultados obtidos podem auxiliar os profissionais que trabalham com terapia nutricional na indicação do tratamento com emulsão lipídica mais indicado para o paciente, tendo em vista a recuperação do aporte calórico podendo levar a melhora de seu quadro clínico.

Por fim, conclui-se também que os lipídios, especialmente e os ácidos graxos ômega-3 e ômega-6, são fundamentais para o desenvolvimento sadio dos pacientes e melhora da qualidade de vida, visto que colaboram para a manutenção das funções cerebrais, desenvolvimento das células, diminuição da pressão sanguínea, prevenção de enfermidades cardiovasculares, proteção contra feridas e infecções, dentre outros. Assim, o consumo das dietas parenterais que contenham tais AGPs, se torna essencial.

\section{Agradecimentos}

Os autores agradecem o Conselho Nacional de Desenvolvimento Científico e Tecnológico (CNPq) e a Coordenação de Aperfeiçoamento de Pessoal de Nível Superior (CAPES) pelo auxílio financeiro.

\section{Referências}

Adolph, M. (1999). Lipid Emulsions in Parenteral Nutrition. 1-13.

Barbalho, S. M., Bechara, M. D., Quesada, K. R., \& Goulart, R. A. (2011). P a pel dos ácidos g r ax os ôme g a 3 na gr axos ômeg lama tórios r esolução dos processos inf processos inflama lamatórios. 44(3).

Berger, M. M. (2014). The 2013 Arvid Wretlind lecture : Evolving concepts in parenteral nutrition. Clinical Nutrition, 33(4), 563-570.

Berger, M. M., \& Pichard, C. (2022). When is parenteral nutrition indicated ? Journal of Intensive Medicine, November 2021.

Boisramé-helms, J., Toti, F., Hasselmann, M., \& Meziani, F. (2015). Progress in Lipid Research Lipid emulsions for parenteral nutrition in critical illness. JPLR, 60, 1-16.

Calder, P. C. (2012). Omega-3 polyunsaturated fatty acids and inflammatory processes : nutrition or pharmacology?

Calder, P. C., Adolph, M., Deutz, N. E., Grau, T., Innes, J. K., Klek, S., Lev, S., Mayer, K., Michael-titus, A. T., Pradelli, L., Puder, M., Vlaardingerbroek, H., \& Singer, P. (2018). Lipids in the intensive care unit: Recommendations from the ESPEN Expert Group *. Clinical Nutrition, 37(1), 1-18.

Calder, P. C., \& Jensen, G. L. (2010). Lipid emulsions in parenteral nutrition of intensive care patients : current thinking and future directions. $735-749$.

Carpentier, Y. A., Dupont, I. E., \& Sc, M. (2000). Advances in Intravenous Lipid Emulsions. 1493-1497.

Casselman, S., Sc, B., D, N. S. M., Kim, P., Phm, B. S., D, D. A. M., Sc, M., Lu, Z., Ph, D., Lou, W., Ph, D., Ma, D., Ph, D., \& D, J. P. A. M. (2021). Olive oil À based lipid emulsion is noninferior to soybean oil À based lipid emulsion in the acute care setting: A double-blind randomized controlled trial. Nutrition, 89, 111283.

Chen, W., \& Yeh, S. (2003). Effects of Fish Oil in Parenteral Nutrition. 9007(02).

Cristina, D. (2009). Terapia nutricional parenteral (tnp). 1-12.

Gavahian, M., Mousavi, A., Lorenzo, J. M., Munekata, P. E. S., Garcia-mantrana, I., Carmen, M., Meléndez-martínez, A. J., \& Barba, F. J. (2019). Trends in Food Science \& Technology Health bene fi ts of olive oil and its components: Impacts on gut microbiota antioxidant activities , and prevention of noncommunicable diseases. Trends in Food Science \& Technology, 88(February), 220-227.

Geert, C. (2007). Immune modulation by parenteral lipid emulsions 1, 2.

Hewavitharana, G. G., Perera, D. N., Navaratne, S. B., \& Wickramasinghe, I. (2020). Extraction methods of fat from food samples and preparation of fatty acid methyl esters for gas chromatography : A review. Arabian Journal of Chemistry, 13(8), 6865-6875.

Itzhaki, M. H., \& Singer, P. (2020). Advances in Medical Nutrition Therapy : Parenteral Nutrition. 1-12.

Klek, S. (2016). Omega-3 Fatty Acids in Modern Parenteral Nutrition : A Review of the Current Evidence. 1-4.

Kojic, D., Feuersenger, A., Schneid, C., Stehle, P., Stover, J., Scheiner, D., \& Westphal, M. (2017). Clinical Nutrition ESPEN Glutamine dipeptidesupplemented parenteral nutrition improves the clinical outcomes of critically ill patients : A systematic evaluation of randomised controlled trials. 17. 
Krohn, K., \& Koletzko, B. (2006). Parenteral lipid emulsions in paediatrics. 319-323.

Martindale, R. G., Berlana, D., Boullata, J. I., Cai, W., Calder, P. C., Deshpande, G. H., Evans, D., \& Garcia-de-lorenzo, A. (2020). Summary of Proceedings and Expert Consensus Statements From the International Summit “ Lipids in Parenteral Nutrition .” 44.

Novak, F., Vecka, M., Meisnerova, E., Sevela, S., Vavrova, L., Rychlikova, J., Dolezalova, L., Myslivcova, D., Zak, A., Vitek, L., \& Novakova, O. (2019). Fish oil supplementation with various lipid emulsions suppresses in vitro cytokine release in home parenteral nutrition patients : a crossover study. Nutrition Research, 72, 70-79.

Perini, L., Stevanato, B., Cyl, S., Eliete, J., Visentainer, L., Dalalio, O., Matshushita, M., Evel, N., Estadual, U., \& Estadual, U. (2010). Ácidos graxos poliinsaturados n-3 e n-6: metabolismo em mamiferos e resposta imune. 23(6), 1075-1086.

Ramalho, H. F., \& Suarez, P. A. Z. (2013). A Qu??mica dos ??leos e gorduras e seus processos de extração e refino. Revista Virtual de Quimica, 5(1), 2-15.

Raman, M., Almutairdi, A., Mulesa, L., Alberda, C., Beattie, C., \& Gramlich, L. (2017). Parenteral Nutrition and Lipids. 1-11.

Rousseaux, M. C., Cherbiy-hoffmann, S. U., Hall, A. J., Searles, P. S., Regional, C., Científicas, D. I., La, T. De, La, R. C. De, \& Ríos, E. (2020). Scientia Horticulturae Fatty acid composition of olive oil in response to fruit canopy position and artificial shading. Scientia Horticulturae, $271,109477$.

Singer, P., Berger, M. M., Berghe, G. Van Den, Biolo, G., Calder, P., Forbes, A., Griffiths, R., Kreyman, G., Leverve, X., \& Pichard, C. (2009). ESPEN Guidelines on Parenteral Nutrition : Intensive care. Clinical Nutrition, 28(4), 387-400.

Singer, P., Reintam, A., Berger, M. M., Alhazzani, W., Calder, P. C., Casaer, M. P., Hiesmayr, M., Mayer, K., Carlos, J., Pichard, C., Preiser, J., Zanten, A. R. H. Van, Oczkowski, S., Szczeklik, W., \& Bischoff, S. C. (2019). ESPEN Guideline ESPEN guideline on clinical nutrition in the intensive care unit. Clinical Nutrition, 38(1), 48-79.

Souza, N. E., \& Visentainer, J. V. (2006). Colesterol da Mesa ao Corpo.Varela.

Torrinhas, R. S., \& Waitzberg, D. L. (2016). Parenteral Nutrition. In Encyclopedia of Food and Health. Elsevier Ltd.

Versleijen, M., Roelofs, H., Preijers, F., Roos, D., \& Wanten, G. (2005). Parenteral lipids modulate leukocyte phenotypes in whole blood, depending on their fatty acid composition. 822-829.

Zhao, Q., Xu, Y., \& Liu, Y. (2022). Food Hydrocolloids Soybean oil bodies : A review on composition, properties , food applications , and future research aspects. Food Hydrocolloids, 124(PA), 107296. 